\title{
A Novel Application of System Survival Signature in Reliability Assessment of Offshore Structures
}

\author{
Tobias-Emanuel Regenhardt ${ }^{1}$, Md Samdani Azad ${ }^{2}$ Wonsiri Punurai ${ }^{2}$, and \\ Michael Beer ${ }^{1}$ \\ 1 Institute for Risk and Reliability, Leibniz University Hanover, \\ Callinstrasse 34, 30167 Hanover, \\ regenhardt@irz.uni-hannover.de \\ 2 Department of Civil and Environmental Engineering, Mahidol University, \\ 25/25 Putthamonthon Nakhorn Pathom 73170, Thailand, \\ mdsaamdani.aza@student.mahidol.ac.th
}

\begin{abstract}
Offshore platforms are large structures consisting of a large number of components of various types. Thus a variety of methods are usually necessary to assess the structural reliability of these structures, ranging from Finite-Elements-methods to Monte-Carlo-Simulations. However, often reliability information is only available for the members and not for the overall, complex, system. The recently introduced survival signature provides a way to separate the structural analysis from the behaviour of the individual members. Thus it is then possible to use structural reliability methods to obtain information about how the failure of several constituent members of the offshore platform leads to overall system failure. This way it is possible to separate the structural from time-dependent information, allowing flexible and computationally efficient computation of reliability predictions.
\end{abstract}

Keywords: structural reliability, offshore platforms, survival signature, system reliability

\section{Introduction}

Offshore jacket platforms are generally used for oil and gas production in shallow and intermediate water depths. Adequate performance of the platforms is ensured by designing for a service life. However, a large numbers of these steel structures are operating exceeded their design life due to high cost of replacement. Consequently, the safety of these offshore platforms creates strong reasons to develop effective methods for the reliability assessment.

For large offshore structures, reliability measures usually concern the structural reliability under the impact of external influences such as fatigue, and corrosion environment. As structural reliability concerns the behavior of an object under physical conditions, a safety assessment should prove that the risk of structural failure is acceptable. The standard methods give some indications, 
such as design code, reserve strength ratio, and a probabilistic value. Design codes are claimed to be very conservative, as more knowledge of the structure is gained through some years after design thereby leading to more accurate analysis results. Methods based on reserve strength ratio can provide insight into the reserve strength of a structure [12]. The reserve strength ratio (RSR) can be obtained from the ratio of ultimate load capacity of the structure divided by the 100 year design load. But it will not cover possible failure modes that could happen to the structure as it provides information regarding the global failure phenomenon as well. Structural reliability methods typically account for the capacity versus loading, particularly deal with uncertainties of structural loads and their effects as well as resistance [14].

Reliability theories basically developed from the concepts of uncertainties (wind, wave and earthquake). An incremental loading approach till the ultimate capacity was conducted for structural reliability is delineated. However, the structural reliability methods are not sufficient measures as they are not consistent with the derivation of the reliability target levels. This is because the reliability assessments deliberates the reliability considering the intensity of environmental conditions (Loads, Corrosion) but not give enough information over time rather these provide information over fixed time. To reduce risk, a better approach is to consider all functional parts of the structure, if present (facilities on offshore platforms, the connections between platforms, pipes, dominant failure modes etc.), exploring patterns and inter-relationships within subsystems and seeing undesired events as the products of the working of the system. Some conventional tools have been used including Failure Tree Analysis (FTA) [11], Failure Mode and Effects Analysis (FMEA) [9], recently, researchers are paying more attention to the statistical techniques. For instance, grey correlation analysis [2], Bayesian Probability [19], Neural network [21], Fuzzy logic evaluation $[8,20]$ and survival signature computing [4] have been applied to the risk assessments in engineering and related fields.

The aim of this paper is to contribute to offshore reliability assessment by using the recently developed survival signature formalism [4]. With this formalism it is possible to predict the reliability of a complex system (as, in this case, an offshore platform) from knowledge about the individual constituents (the platform members). With this, it is possible to divide the reliability assessment into two individual steps. Firstly the system structural system is analysed. This is achieved through finding the combinations of failing members that lead to total failure of the whole offshore platform. Secondly, the information about the members' reliability over time is multiplied with the corresponding entries of the survival signature to predict the overall reliability. 


\section{The Survival Signature}

\subsection{System Reliability Applying the Survival Signature}

The state of a any system set together of $m$ independent and different components can be represented by a state vector $\underline{x} \in\{0,1\}^{m}$ with $x_{i}=0$ denoting a dysfunctional and $x_{i}=1$ a functional component $i$.

The global structure function $\varphi:\{0,1\}^{m} \rightarrow\{0,1\}$ contains information whether the system is in a working state $(\varphi=1)$ or not $(\varphi=0)$ for any possible $\underline{x}$. Usually the observed systems are restricted to coherent systems. This refers to systems with $\varphi$ not decreasing in any dimension of $\underline{x}$. This assumption is sound as most common systems are not becoming dysfunctional while gaining more functional components. Two additional assumptions are $\varphi(\underline{0})=0$ and $\varphi(\underline{1})=1$. These are intuitive, yet not necessary. However, in this paper the monotonicity of the system is assumed and thus these two conditions and the coherency of the system are assumed as well.

For more complex systems, every component belongs to one of $K$ different types, while each set of components of type $k \in\{1,2, \ldots, K\}$ consists of $m_{k}$ elements and the sum of all $m_{k}$ equals the number of components $\sum_{k=1}^{K}=m$ . The amount of functional components of type $k$ present in the system are denoted as $l_{k}$. This leads to $\left(\begin{array}{c}l_{k} \\ m_{k}\end{array}\right)$ possible combinations of component type $k$ under the assumption of independent failure of all components. Then the set $S(\underline{l})$ is the collection of all state vectors that fulfil the condition that $\underline{l}=\left(l_{1}, l_{2}, \ldots, l_{k}\right)$ components are working. The system's survival signature $\Phi$ is now defined as the probability that the system is functional if exactly $l_{k}$ components of type $k$ are functional [23]. The survival signature is an array of $K$ dimensions with $m_{k}+1$ entries in each dimension (including the case that none of the components of that type function). For components with exchangeable random failure times the survival signature is given by

$$
\Phi\left(l_{1}, l 2, . ., l_{K}\right)=\left[\prod_{i=1}^{K}\left(\begin{array}{c}
l_{k} \\
m_{k}
\end{array}\right)\right]^{-1} \times \sum_{\underline{x} \in S(\underline{l})} \varphi(\underline{x}) .
$$

The survival signature can be applied to the computation of the survival function of the system: $P\left(T_{S}>t\right)$. It provides the probability that a random failure time $T_{S}$ of the system follows a specific point in time $t$. This provides the reliability of the system in time. Under the assumption of the failure times of the components being independently and identically distributed (iid), with respect to a known cumulative distribution function [15]. $F_{k}(t)$, the survival function of the system observed is found to be

$$
P\left(T_{S}>t\right)=\sum_{l_{1}=0}^{m_{0}} \ldots \sum_{l_{k}=0}^{m_{k}}\left[\Phi(\underline{l}) \times \prod_{k=1}^{K}\left(\begin{array}{c}
l_{k} \\
m_{k}
\end{array}\right) F_{k}(t)^{m_{k}-l_{k}}\left[1-F_{k}(t)\right]^{l_{k}}\right] .
$$

Eq. (1) and (2) indicate show that - for exact computation - many different states need to be evaluated and that the size of the survival signature itself is 
growing multilinearly. However, for small- and medium-sized systems the survival signature can be calculated exactly or by Monte Carlo Simulation methods. The use of signature frameworks can be useful in several ways. It seperates the information about the system in two subproblems to be solved. If the system structure is unchanged, the survival signature stays the same even if the behaviour of the components changes. Thus testing of components in simulations is computationally efficient. Additionally, additions to the framework can easily be done, for example in case of repairable systems [22] or in case of components with multiple states [5].

\subsection{Obtaining the Survival Signature from Structural Simulations}

The calculation of the $\Phi$ necessitate knowledge about the behaviour of the system under failure of the components. Usually due to complexity, an explicit global structure function is often not given. Instead, the states of the system can be evaluated by various means, including reliability block diagrams and cut-sets, binary decision diagrams, and failure tree analysis [17].

With reliability block diagrams, it is simple to visualize the behaviour of small systems. However, the search of cut-sets in a block diagram is NP-hard and can be very time consuming [18]. Binary decision diagrams can provide fast means to calculate the survival signature once the decision diagram data structure is available. However, the calculation of the decision diagram is also dependent on finding the cut-sets of the system and can be, inherently, slow.

For structural reliability, one is usually concerned with the behaviour of a structure under load. Thus the interaction of the various components is not modelled in any way described above - instead, the structure is modelled and analysed in frameworks of mechanical simulation methods (commonly, finite elements methods) concerned with the actual physical behaviour.

In this work, a bridge over this gap is presented. A large structure consists of several, possibly redundant, components. This means that the system might still be operational after the failure of some of the components. Thus, structural simulation can show various failure modes of the system under load. If a structural simulation of the structure results in a failing component, the structure is updated and the simulation started again. This is repeated until the simulation results show that the structure is failing in total. All failed components until this point are saved in a failure mode. By variation of the load parameters, all components prone to failure are identified and several failure modes are identified. These failure modes can be used as cut-sets in computation of the survival signature. Equation (1) can be evaluated using these cut-sets to compute the values of $\varphi(\underline{x})$ for all $\underline{x}$ (exact computation) or a representative sample (Monte Carlo Simulation). In this study, the Monte-Carlo approach was used as the amount of combinations to be checked is of medium size. The largest amount of combinations that is possible is for the entry placed directly in the middle of the array $\left(\left(\begin{array}{l}3 \\ 2\end{array}\right)^{3} \cdot\left(\begin{array}{l}8 \\ 4\end{array}\right)=1890\right)$. Thus a sample size of 2000 samples was used. 


\section{Reliability of an Offshore Jacket Platform}

\subsection{Structural Model}

The jacket platform is taken from [16]. The Jacket is designed for shallow water depth of approximately $65.31 \mathrm{~m}$. It is a 4-legged jacket containing pile inside the legs. The jacket is modeled as $2 \times 2$ square grid. The overall dimensions are $8 \times 8 \mathrm{~m}$ at top elevation and $21.76 \times 21.76 \mathrm{~m}$ at the mud line. The total height is $81 \mathrm{~m}$. Two types of bracings are used named as horizontal bracings and vertical bracings. The horizontal bracings are installed at five levels. The vertical bracings are provided as single bracings till the bottom level. At the bottom level, it was provided as K-bracings to impart more stiffness and reduce buckling. The jacket support/foundation is modelled as fixed support system. The jacket is modelled in SAP2000 as shown in Fig. 1. Member properties of the jacket are also taken from [16]. The top mass of the oil and gas platform is simplified as a lumped

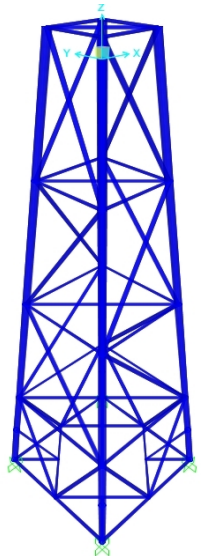

(a)

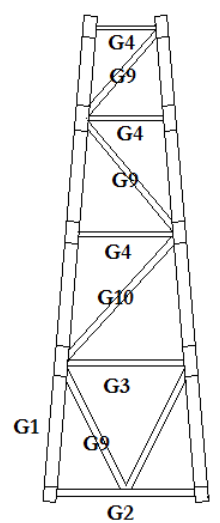

(b)

Fig. 1. (a) Three-dimensional model of the jacket (b) Grouping of the components

mass for the easiness finite element modelling. The total weight of the topside is assumed as 1250 tons, which is equally applied over four legs where each leg, is carrying 312.5 tons at the top nodes of the jacket structures platform.

\subsection{Generation of Failure Tress}

Non-linear static (pushover) analysis is performed to understand the behaviour of structure against lateral load pattern following the procedure of FEMA356 [6] and [9]. The behaviour of the force displacement curve can be observed from the analysis as well. In this step, the structure is incrementally loaded over its 
yielding capacity and to observe the ultimate load level. The failed elements are recorded up to the ultimate load level. Here the damage level is not considered because the aim is to grasp the failure behaviour of members up to ultimate load level. Here the term failure behaviour is defined as how the member fails chronologically and which member is followed by another member. Load has been applied along three different directions comprising of $0^{\circ}, 90^{\circ}$ and $45^{\circ}$. The typical pushover curve is shown in Fig. 2 which is adopted from FEMA356 [6] and the ultimate load level is the point ' $\mathrm{C}$ '. The failure tree can be observed in Fig. 3.

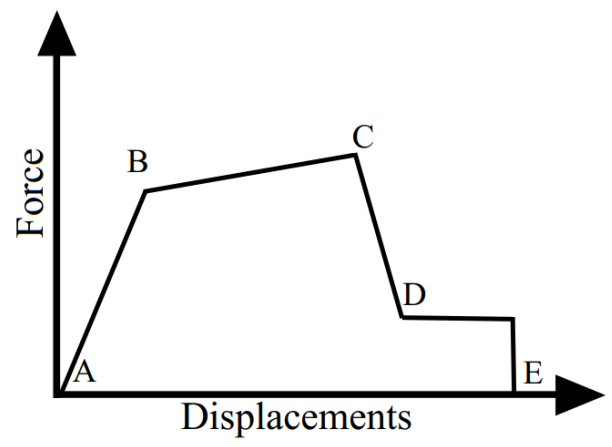

Fig. 2. Non-linear force curve

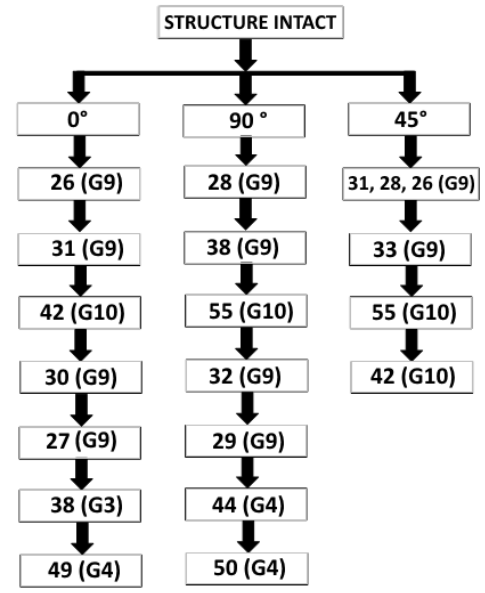

Fig. 3. Failure tree of the offshore platform under various loads 
The tree is generated for three different load directions. For zero degree of direction, the first damage is observed in member 26. After that, this member is followed by members $31,41,30,27,38$, and 49 . When the damage initiates in member 49, the load level reached the ultimate level. For 90 degree direction, the first damage can be noted in member 28. The other members are 38, 55, 32, 29, 44 and 50. In case of 45 degree loading direction, the first damage is detected in 3 members in parallel labelled as 31, 28 and 26. The reason behind this is that the load is equally distributed over 2 orthogonal directions which caused three members to fail at once.

\subsection{CDF of Component Types}

After the successful identification of the failure-prone members of the offshore platform, it is necessary to choose proper cumulative distribution functions (CDF) for the calculation of the survival function. In this study, the components are the failure-prone members of the platform grouped in four different types according to tube total diameter $\left(d_{i}\right)$ and wall thickness $\left(\tau_{i}\right)$. These groups can be found in Table 1.

Table 1: Overview of the failure prone component types

\begin{tabular}{lcccc} 
Group & $d_{i}[\mathrm{~m}]$ & $\tau_{i}[\mathrm{~m}]$ & $\mu_{i}$ & $\sigma_{i}$ \\
\hline \hline 3 & 0.013 & 0.406 & 1.000 & 0.895 \\
4 & 0.010 & 0.356 & 0.769 & 0.805 \\
9 & 0.013 & 0.550 & 1.000 & 1.000 \\
10 & 0.019 & 0.559 & 1.462 & 1.008 \\
\hline
\end{tabular}

As many environmental factors influence each platform individually (corrosion rates, ocean movement, fatigue, and usage of protection measurements against these), in this study a general log-normal distribution is assumed. The two form factors (mean $\mu$ and standard deviation $(\sigma)$ are under strong influence of the environment and the individual situation the platform is modelled or investigated in.

Thus, as a proof of concept, the mean time of failure and standard deviation is assumed to be of unity for the component group 3. To properly scale the other groups' parameters, in first order approximation, the mean follows $\mu_{i} \sim \tau_{i}$ and $\sigma_{i} \sim \sqrt{d_{i}}$, respectively. In an applicated situation, the distribution of failure times can be obtained in dependence of the individual situation. As corrosion is one of the most important influences to offshore reliability, the corrosion rate can be measured over time and compared with the thickness of the affected members to estimate the probability that a member has corroded to an unstable state at a given time. Additionally the corrosion can be modelled if precise measurements and knowledge about the situation is available (e.g. salinity, $\mathrm{pH}$ value and corrosion countermeasures). Also the influence of fatigue can be taken into account. In the most optimal case, a thorough study of the material behaviour can be 
done on similar structures already present. The repair and maintenance rates can be easily adopted to estimate values for the mean values of the failure times of a certain component type (standard deviation is then just a matter of how the data spreads).

Measurements concerning the structural properties that relate to time-dependent reliability are, as all measurements, to some degree imprecise. Additionally, many aspects of the temporal behaviour of the structural elements is only known to a certain degree beforehand. Thus imprecision has to be taken in account. To demonstrate imprecise probabilities in this case, the survival function can be computed by using an upper and lower bounds $\left(\bar{F}_{k}(t)\right.$ and $\left.\underline{F}_{k}(t)\right)$ for the CDF together with the survival signature formalism $[4]$

$$
\bar{P}\left(T_{S}>t\right)=\sum_{l_{1}=0}^{m_{0}} \ldots \sum_{l_{k}=0}^{m_{k}}\left[\Phi(\underline{l}) \times \prod_{k=1}^{K}\left(\begin{array}{c}
l_{k} \\
m_{k}
\end{array}\right) \bar{F}_{k}(t)^{m_{k}-l_{k}}\left[1-\bar{F}_{k}(t)\right]^{l_{k}}\right],
$$

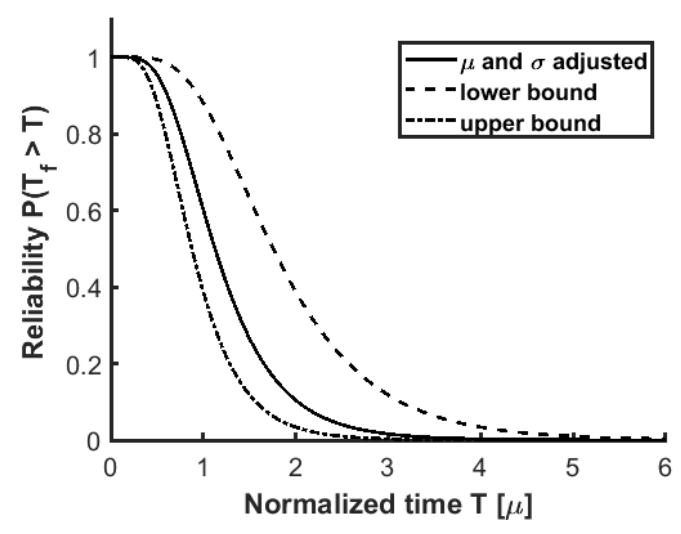

Fig. 4. Survival functions of the system

In this example, the highest and lowest value of $\mu_{i}$ was chosen to generate upper and lower bounds for the CDF and thus to generate a bounding p-box for the time-dependent reliability. The corresponding survival functions can be found in Fig. 4. The survival functions not only show the expected behaviour in reliability for the whole system, it becomes also clear that using only slightly different probability distributions can have grave impact on the long term reliability of the system. 


\section{Summary}

This study shows that structural information can be obtained in order to apply the survival signature formalism - which originates from system reliability - to structural problems.

With the introduced method, it was possible to identify the members of the offshore structure whose failure leads to overall system failure. It becomes clear that under load only a selected few of the components of the platform are relevant to the failure modes of the platform. This reduces the amount of components to be considered in the system reliability measurements and makes the analysis using system reliability methods feasible. Originating from observations made during the initial state of the system a reasonable prediction of the behaviour in time can be made. For example, a maintenance cycle can be defined by setting a certain value for the reliability: as soon as the reliability is less than the defined value, maintenance has to occur.

However, individual temporal behaviour is highly dependent on the environmental situation and has to be implemented carefully. The impact of imprecise probabilities is to be taken into account properly in the future.

\section{Acknowledgements}

This project has received funding from the European Union's Horizon 2020 research and innovation under the Marie Skodowska-Curie grant agreement No. 730888. This work was also funded by the Deutsche Forschungsgemeinschaft (DFG, German Research Foundation) grants BE 2570/3-1 and BR 5446/1-1 as part of the project 'Efficient Reliability Analysis of Complex Systems'.

\section{References}

1. Bai, Y., Yan, H., Cao, Y., Kim, Y., Yang, Y., Jian H.: Time-dependent Reliability Assessment of Offshore Jacket Platforms. Ships and Offshore Structures. 11 (6), 591-602 (2015)

2. Bao, X., Cao, A., Qin, F.: Safety Assessment of an Aging Offshore Jacket Platform by Integrating Analytic Hierarchy Process and Grey Clustering Method. Preprints 2016, $2016080182(2016)$

3. Bilionis, D. V., Vamvatsikos, D.: Fatigue Analysis of an Offshore Wind Turbine in Meditteranean Sea under a Probabilistic Framework. VI International Conference on Computational Methods in Marine Engineering, MARINE (2015)

4. Coolen, P. A., Coolen-Maturi, T., Aslett, L., Gero, W.: Imprecise System Reliability using the Survival Signature. ICAMER'16: Proceedings of the 1st International Conference on Applied Mathematics in Engineering and Reliability(2016)

5. Eryilmaz, S., Tuncel, A.: Generalizing the Survival Signature to Unrepairable Homogeneous MultiState Systems. Naval Research Logistics. 63 (8), 593-599 (2016).

6. Federal Emergency Management Agency: Prestandard and Commentary for the Seismic Rehabilitation of Buildings, chapter 3 (2000) 
7. Golafshania, A. A., Tabeshpourb, M. R., Komachia, Y.: FEMA approaches in seismic assessment of jacket platforms. Journal of Constructional Steel Research. 65, 1979-1986 (2009)

8. Kang, H., Han, J., Zhou, P.: Risk Assessment for Offshore Jacket Platform Based on Fuzzy Probabilistic Influence Diagram. Proceedings of 16th International Offshore and Polar Engineering Conference, San Francisco, CA, USA (2006)

9. Kim, D., Ok, S., Song, J., Koh, H.: System reliability analysis using dominant failure modes identified by selective searching technique. Reliability Engineering and System Safety. 119, 316-331 (2013)

10. El-Din, M. N., Kim, J.: Seismic Performance Evaluation and Retrofit of Fixed Jacket Offshore Platform Structures. Journal of Performance of Constructed Facilities, 29(4), 040114099 (2015)

11. Kurian, V. J., Wahab, M. M. A., Kheang, T. S., Liew, M. S.: System Reliability of Existing Jacket Platform in Malaysian Water. Applied Mechanics and Materials. 567, 316-331 307-312 (2013)

12. Majid, W. M. b. W. A., Hashim, A. R. b. H., Embong, M. b.: Determination of Structural Reserve Strength Ratio (RSR) of an Existing Offshore Structure. The Eighth International Offshore and Polar Engineering Conference (1998)

13. Melchers, R. E., Beck, A. T.: Structural Reliability Analysis and Prediction. Wiley, Hoboken (2017)

14. Moses, F., Stahl, B.: Reliability Analysis Format for Offshore Structures. Journal of Petroleum Technology, 31 (03), 347 (1979)

15. Patelli, E., Feng, G., Coolen, F.P.A., Coolen-Maturi,T.: Simulation Methods for System Reliability Using the Survival Signature. Reliability Engineering \& System Safety. 167, 327-337 (2017)

16. Punurai, W., Azad, M. S., Pholdee, N., Sinsabvarodom, C.: Adaptive metaheuristic to predict dent depth damage in the fixed offshore structures. Accepted paper, European Safety and Reliability Conference (2018)

17. Reed, S.: An efficient algorithm for exact computation of system and survival signatures using binary decision diagrams. Reliability Engineering \& System Safety. 165, 257-267 (2017)

18. Supplement information to [17] https://github.com/sean-reed/bdd-signatures

19. Ren, J., Wang., J., Jenkinson, I., Xu, D. L., Yang, J. B.: A Bayesian Network Approach for Offshore Risk Analysis through Linguistic Variables. China Ocean Engineering, 21 (3), 371-388 (2007)

20. Ren, J., Jenkinson, I., Wang, J., Xu, D. L., Yang, J. B.: An Offshore Risk Analysis Method Using Fuzzy Bayesian Network. J. Offshore Mech. Arct. Eng 131 (4), (2009)

21. Uddin, Md. A., Jameel, M., Razak, H. A.: An Offshore Risk Analysis Method Using Fuzzy Bayesian Network. Indian Journal of Geo-Marine Science. 44 (3) (2015)

22. Walls, L., Revie, M., Bedford, T.: Risk, Reliability and Safety: Innovating Theory and Practice. CRC Press, Boca Raton, 2017

23. Zamojski,W., Mazurkiewicz, J., Sugier, J., Walkowiak, T., Kacprzyk, J.: Complex Systems and Dependability. Springer, Berlin (2012) 